\title{
Work-function changes due to surface anisotropy and imperfections
}

\author{
A. N. Andriotis \\ Theoretical and Physical Chemistry Institute, National Hellenic Research Foundation, \\ 48, Vassileos Constantinou Avenue, Athens 116/35, Greece \\ (Received 9 August 1984; revised manuscript received 5 April 1985)
}

\begin{abstract}
Our previously reported theory for semi-infinite metals, which incorporates lattice effects in a direct way within a self-consistent scheme of the type of Hohenberg and Kohn, is used here for calculating the variation of the work function of the $\mathrm{Na}(001)$ surface during the process of building up a new (001) lattice plane on top of its surface plane. It is shown that this variation follows the trend exhibited during alkali chemisorption onto a metal surface. In addition, more results are reported, indicating the effect of the lattice anisotropy on the work function of the alkali metals.
\end{abstract}

\section{INTRODUCTION}

In the process of calculating the work function of simple metals it is usually assumed that the crystal surface is a lattice plane with a positive-ion concentration and positioning defined via the bulk-crystal parameters. ${ }^{1-5}$ Under these assumptions the bulk crystal is continued up to the surface lattice plane which bounds the crystal from the vacuum region. However, experimental and theoretical studies have shown that these two conditions are not fulfilled in general. The positioning of the surface lattice plane is not the one which is expected from the lattice constant of the (bulk) crystal. Instead the surface lattice plane is relaxed to a position which satisfies the energetics of the system..$^{6-10}$ On the other hand, the surface lattice plane may exhibit other defects which destroy the perfectness of the surface lattice plane as compared to a bulk lattice plane. The term "perfectness" of the surface plane is associated here with the concentration of positive ions on this plane as this concentration is compared with that of the bulk lattice plane. Thus an $(h k l)$ surface plane specified by the Miller indexes $h k l$ is defined, here, as perfect if the concentration of the positive ions on this plane is the same as that of a bulk $(h k l)$ crystal plane. No other type of defects are considered.

For simplicity it is assumed throughout this work that the semi-infinite crystal under consideration possesses a Bravais lattice and all crystal planes parallel to the surface are topologically equivalent.

The surface relaxation and the surface perfectness affect the electronic configuration near the surface of the metal and they are exhibited in the surface properties as the work function and the surface energy of the metal. While surface perfectness is of considerable importance, ${ }^{11}$ the surface relaxation may be neglected in work-function and surface-energy calculations. ${ }^{12}$

Both the effects of the surface perfectness and surface relaxation may be estimated within the jellium-model approximation. In the case of surface relaxation, the surface region of the crystal is approximated by a jellium slab in contact with the bulk jellium. The positive charge density of the jellium slab depends on the relaxation and is specified by the condition of charge conservation. Similarly, the imperfect surface plane of the crystal may be approximated by a jellium slab in contact with the bulk jellium. The positive charge of the jellium slab which represents the imperfect surface plane depends on the concentration of the positive ions in this plane. The jellium approximation of an imperfect surface is similar to the jellium approximation proposed by $\mathrm{Lang}^{13}$ in studying the dependence of the work function of a metal upon alkali chemisorption.

The jellium-model approximation also proved useful in studying the effect of crystal anisotropy on the work function. ${ }^{14}$ In the simplest case, in the jellium approximation to a semi-infinite crystal in the $\langle h k l\rangle$ orientation, the jellium edge is assumed to be located within the vacuum region at half the interplane distance, $c_{\langle h k l\rangle}$, of the $(h k l)$ lattice planes. This assumption is helpful in obtaining the trends of the anisotropy effects. However, for quantitative results the crystal anisotropy requires the explicit inclusion of the lattice geometry in describing the singleelectron potentials and wave functions of a metallic system. The earlier approaches utilize the jellium approximation as the zeroth-order approximation (ZOA) to which the lattice effects are added either perturbatively ${ }^{2,3}$ or/and variationally.,5 Although these approximations resulted in theoretical estimations for the work function and the surface energy which agree with the experimental ones, they result in electron density profiles which are of jellium type and exhibit neither the crystal periodicity nor the trends found in complete three-dimensional calculations. ${ }^{15}$ In addition to these drawbacks, the jellium-based calculations fail to include band-structure effects due to the character of these models.

In previous papers ${ }^{16,17}$ we proposed a model approximation which eliminates the above-mentioned drawbacks of the jellium-based approaches. The proposed model retains the simplicity of the jellium approximation in the sense that it is a one-dimensional problem. In addition to this it allows inclusion of band-structure effects, since the band structure along the direction $\langle h k l\rangle$, which is perpendicular to the surface, is taken into account in the process of calculating the single-electron wave functions of the metal. Due to its nature this model introduces the crystal geometry directly within the single-electron 
Schrödinger equation, avoiding in this way any perturbative or variational treatment of the lattice effects. Our model is the zeroth-order approximation of a general three-dimensional model solution ${ }^{16}$ which utilizes the propagation matrix technique ${ }^{18}$ in solving the Schrödinger equation for the free electrons of a metal whose ionelectron interactions are described by a local pseudopotential. The model solution follows the self-consistent scheme of Hohenberg and Kohn. ${ }^{19}$

In the process of solving this three-dimensional model, we expand the single-electron wave functions and potentials in Fourier series with respect to the two-dimensional reciprocal space of the surface lattice plane. The Fourier coefficients are coordinate dependent. The zeroth-order approximation to this three-dimensional model solution retains only the zeroth-order Fourier term in the Fourier series expansions. Thus the ZOA model solution becomes a one-dimensional problem. This approximation was shown to be very good in the case of simple metals. ${ }^{16} \mathrm{We}$ used this ZOA model to calculate the changes in the work function ${ }^{16}$ and the surface energy ${ }^{20}$ due to the crystal anisotropy. Also, it was found that the electron density profiles obtained within the ZOA model solution follow the trends found by more complicated calculations. ${ }^{15}$

The physical consequence of the ZOA is that within this approximation the positive charge distribution, $\rho_{+}(z)$, of the positive ions of the metal takes the form of a multiplanar charge distribution

$$
\rho_{+}(z)=\sum_{l=0}^{\infty} \frac{2 \pi}{a} \delta\left(z-z_{l}\right)
$$

in planes parallel to the surface (Fig. 1). In Eq. (1), $z_{1}$ denotes the position of the $(h k l)$ planes, i.e., $z_{1}=-l c\langle h k l\rangle$, where $l$ is an integer $\geq 0$. The $a$ in Eq. (1) denotes the area of the unit cell on the $(h k l)$ lattice plane. For completeness we review the formalism of the proposed model and then we apply it to study the effect of the surface perfectness on the electronic properties of a metal surface.

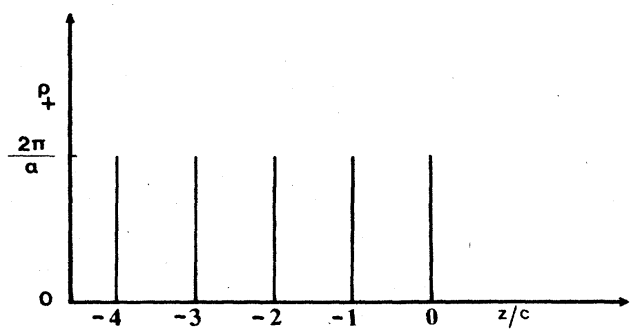

FIG. 1. In the zeroth-order approximation (see text), the charge of the positive ions of a semi-infinite metal exhibiting the $\langle h k l\rangle$ orientation, is smeared cut along the $(h k l)$ lattice planes. The (positive) charge density $\rho_{+}$(per unit area) is $2 \pi / \alpha$ where $a$ is the area of the $2 \mathrm{D}$ unit cell on the ( $h k l)$ lattice plane. The interplane distance is denoted by $c$. The surface plane is located at $z=0$. It is assumed that the semi-infinite crystal occupies the negative $z$ space, possesses a Bravais lattice and all crystal planes parallel to the surface are topologically equivalent.

\section{REVIEW OF THE MODEL}

The symmetry of the system and in particular the twodimensional (2D) periodicity on planes parallel to the surface, allows the expansion of single-electron potentials and wave functions in Fourier series with respect to the $2 \mathrm{D}$ reciprocal lattice of the surface plane. Assuming that the semi-infinite crystal occupies the negative $z$ space, the Fourier coefficients in the Fourier series expansions appear $z$-coordinate dependent.

The ion-electron interaction is described within the local pseudopotential approximation of Ashcroft's type. ${ }^{21}$

The zeroth-order Fourier coefficients of the pseudoion-electron interaction $V^{i-e}(\mathbf{r})$ and the Hartree term $V^{e-e}(\mathbf{r})$ of the electron-electron interactions are not defined separately. ${ }^{16}$ This is due to the fact that these zeroth-order coefficients exhibit a singularity with respect to the 2D reciprocal-lattice vectors $q$ which is of $|q|^{-1}$ type. However, it was shown ${ }^{16,17}$ that a zeroth-order term $V_{0}(z)$ of the sum of the two potentials may be defined as

$$
V_{0}(z)=\lim _{\mathrm{q} \rightarrow 0}\left[V_{\mathrm{q}}^{i-e}(z)+V_{\mathrm{q}}^{e-e}(z)\right],
$$

where $f_{\mathrm{q}}(z)$ denotes the Fourier coefficients of the function $f(\mathbf{r})$. Our approximation to the zeroth-order Fourier coefficients of the exchange and correlation potential $V_{\mathrm{xc}}(z)$ is taken within the local-electron-density form and from Wigner's ${ }^{22}$ expression for the average exchange and correlation energy per electron.

In the ZOA model solution, the Schrödinger equation

$$
-\frac{1}{2} \frac{d^{2} \xi_{q}}{d z^{2}}+\left[V_{0}(z)+V_{\mathrm{xc}}(z)\right] \xi_{q}(z)=E_{q} \xi_{q}(z)
$$

is solved self-consistently for the single-electron wave functions $\xi_{q}(z)$ and the single-electron potentials $V_{0}(z)$ and $V_{\mathrm{xc}}(z)$. The latter are functionals of the electron density $\rho(z)$ given by

$$
\rho(z)=\frac{1}{2 \pi} \sum_{q}\left(k_{F}^{2}-q^{2}\right)\left|\xi_{q}(z)\right|^{2},
$$

where $k_{F}$ is the wave vector corresponding to the Fermi energy $E_{F}=\frac{1}{2} k_{F}^{2}$.

On the one hand, it has been implicitly assumed that the Fermi surface is a sphere of radius $k_{F}$. On the other hand, due to the explicit inclusion of the lattice periodicity (along the $z$ axis), $E_{q}$ is the part of the band structure of the crystal along the $\langle h k l\rangle$ direction, namely the $\langle 001\rangle$ direction in this case. This is due to the fact that $V_{0}(z)$, obtained by the use of Eq. (2), exhibits the periodicity of the crystal along the $z$ axis. This is the advantage of our approach which allows a direct incorporation of lattice effects within the Schrödinger equation. In this way any perturbative or variational treatment of the lattice effects is avoided.

The Schrödinger equation [Eq. (3)] is solved numerically in the bulk and vacuum regions. The solution in the bulk region proceeds (as in the case of the threedimensional model solution) by calculating the propagation matrix which is subsequently diagonalized. The two eigenvalues of the propagation matrix have absolute value equal to one and correspond to two independent propaga- 
ting solutions within the bulk crystal. Then the bulk solution is matched with the surface solution. More details about this approach and the numerical solution may be found in our earlier reports. ${ }^{16,17,20}$

The ZOA model solution was originally used for estimating the effect of crystal anisotropy on the work function and the surface energy of the Na crystal. Some more numerical results are given in Table $I$, which shows the effect of the crystal anisotropy on the work function of the alkali metals. In Fig. 2 the relationship $E_{q}=E(q)$ is shown for the case of $\mathrm{Al}(001)$. It is clear that $E_{q}$ exhibits the band gap of the band structure of Al in the $\langle 001\rangle$ direction ( $\Gamma X$ part of the band).

It should be noted, however, that the ZOA model solution is more applicable to metals for which the Fermi surface (sphere), when projected onto the $2 \mathrm{D}$ reciprocal space of the surface lattice is found to be located approximately within the first 2D Brillouin zone of the 2D reciprocal lattice of the surface plane. In such cases, the assumed approximation $E_{F}=\frac{1}{2} k_{F}^{2}$ is expected to be satisfactory.

In the next section we refer to the use of the present approach in calculating the variation of the work function due to imperfections in the surface plane.

\section{INCORPORATION OF SURFACE PERFECTNESS}

Due to the multiplanar charge distribution this model may also be used to calculate the work-function changes due to ionic chemisorption. ${ }^{17}$ It is only necessary to specify the chemisorbed species and its concentration. Assuming that the chemisorbed ions form a layer parallel to the surface, we can derive an analytical form of the ionelectron interaction for the chemisorbed system. This ion-electron interaction is incorporated within the Schrödinger equation which is solved subsequently. The concentration of the chemisorbed ions may be defined through an effective valency, $Z_{\text {eff }}$, given by

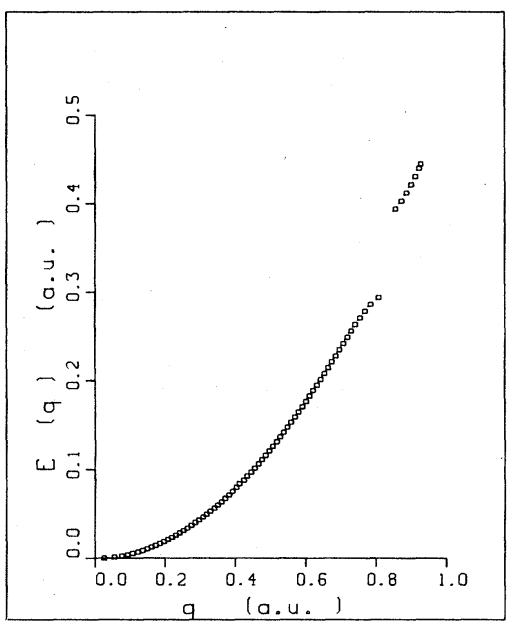

FIG. 2. The $\Gamma X$ part of the occupied band structure $E=E(q)$ of $\mathrm{Al}(100)$ (in the extended-zone scheme) as obtained self-consistently using the zeroth-order model approximation described in the text. The energy values are shifted so that $E(q=0)=0.0$.

$$
Z_{\mathrm{eff}}=\frac{a_{\mathrm{sub}}}{a_{\mathrm{ads}}} Z_{\mathrm{ads}},
$$

where $a_{\text {sub }}$ denotes the area of the two-dimensional unit cell of the surface plane of the substrate, $a_{\text {ads }}$ denotes the area of an average unit cell on the adsorbate layer (which depends on the concentration of the adatoms), and finally $Z_{\text {ads }}$ is the actual valency of the chemisorbed ions. It may be argued that the position $d$ of the chemisorbed layer (from the surface lattice plane) leaves an undetermined parameter. However, as we have shown, ${ }^{26}$ this parameter

TABLE I. Variation of the work function of the alkali metals $\mathrm{Li}, \mathrm{Na}, \mathrm{K}$, and $\mathrm{Rb}$ due to the anisotropy of the crystal, as obtained from self-consistent calculations using the zeroth-order model approximation (see text). The core radii $r_{c}$ describe Ashcroft's (Ref. 21) local pseudopotential used in these calculations. For comparison, results of other calculations are also included.

\begin{tabular}{|c|c|c|c|c|c|c|c|}
\hline \multirow[b]{2}{*}{ Element } & \multirow[b]{2}{*}{$\begin{array}{c}r_{c} \\
\text { (a.u.) }\end{array}$} & \multicolumn{6}{|c|}{ Work function $(\mathrm{eV})$} \\
\hline & & $\begin{array}{l}\text { This work } \\
\text { and } \\
\text { Ref. } 16\end{array}$ & $\begin{array}{c}\text { Kiejna- } \\
\text { Wojciechowski } \\
\text { (Refs. 23, 24) }\end{array}$ & $\begin{array}{l}\text { Bohnen } \\
\text { Ying } \\
\text { (Ref. 15) }\end{array}$ & $\begin{array}{l}\text { Monnier } \\
\text { et al. } \\
\text { (Refs. 4,5) }\end{array}$ & $\begin{array}{l}\text { Lang- } \\
\text { Kohn } \\
\text { (Ref. 3) }\end{array}$ & Expt. $^{\mathrm{a}}$ \\
\hline $\mathrm{Li}(110)$ & 1.06 & 3.77 & $3.31-3.18$ & & 3.5 & 3.55 & 2.32 \\
\hline $\operatorname{Li}(100)$ & & 3.56 & $3.26-3.11$ & & 3.4 & 3.30 & 3.1 \\
\hline $\operatorname{Li}(111)$ & & 3.42 & $3.12-2.96$ & & 3.2 & 3.25 & \\
\hline $\mathrm{Na}(110)$ & 1.67 & 3.22 & $3.06-2.91$ & 3.1 & 3.13 & 3.10 & 2.7 \\
\hline $\mathrm{Na}(100)$ & & 3.08 & $2.94-2.77$ & 2.7 & 2.84 & 2.75 & $2.9^{\mathrm{b}}$ \\
\hline $\mathrm{Na}(111)$ & & 2.82 & $2.73-2.56$ & & 2.76 & 2.65 & \\
\hline $\mathrm{K}(110)$ & 2.14 & 2.76 & $2.75-2.58$ & 3.2 & 2.9 & 2.75 & 2.39 \\
\hline $\mathbf{K}(100)$ & & 2.59 & $2.60-2.43$ & 2.8 & 2.7 & 2.40 & \\
\hline $\mathbf{K}(111)$ & & 2.42 & $2.38-2.21$ & & 2.5 & 2.35 & \\
\hline $\mathrm{Rb}(110)$ & 2.61 & 2.40 & $2.63-2.46$ & 2.6 & 2.9 & 2.20 & 2.21 \\
\hline $\mathrm{Rb}(100)$ & & 2.40 & $2.45-2.28$ & 2.3 & 2.6 & 2.10 & \\
\hline $\mathrm{Rb}(111)$ & & 2.22 & $2.23-2.06$ & & 2.3 & 2.05 & \\
\hline
\end{tabular}

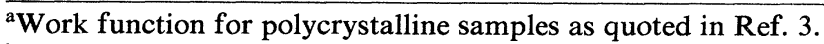

${ }^{b}$ Work function for $\mathrm{Na}(110)$ (Ref. 25). 
is uniquely specified by minimizing the total energy of the chemisorbed system with respect to the position of the adlayer. Numerical results for the systems $\mathrm{Li}^{+} / \mathrm{Na}(100)$ and $\mathrm{Na}^{+} / \mathrm{Al}(100)$ have been reported elsewhere. ${ }^{27}$ In the present work it is assumed that the chemisorbed layer consists of ions of the same type as that of the substrate. Surface relaxation effects are left out so that the chemisorbed layer is positioned at the interplane distance $c_{\langle h k l\rangle}$. Varying the concentration of the ions of the adlayer or equivalently varying the effective valency $Z_{\text {eff }}$, we follow the formation of a new lattice plane on top of the surface of the substrate. For each concentration of the adlayer, Schrödinger's equation is solved self-consistently. Then the obtained electron density profile $\rho(z)$ is used to calculate the work function of the system whose surface plane is an incomplete lattice plane. It should be under- stood, however, that this model may not be appropriate for very low adsorbate concentrations for the same reasons that the corresponding jellium approximation is not applicable. ${ }^{13}$ Our results are presented in the next section.

\section{RESULTS AND DISCUSSION}

Figure 3(a) shows the electron density profile of the $\mathrm{Na}(001)$ crystal which occupies the negative $z$ space. In Figs. 3(b)-3(e) we present the electron density profiles for the semi-infinite $\mathrm{Na}(001)$ crystal whose surface plane has a variable positive-ion concentration. Figure 4 exhibits the variation of the work function of the $\mathrm{Na}(001)$ crystal due to surface imperfectness. In other words, Fig. 4
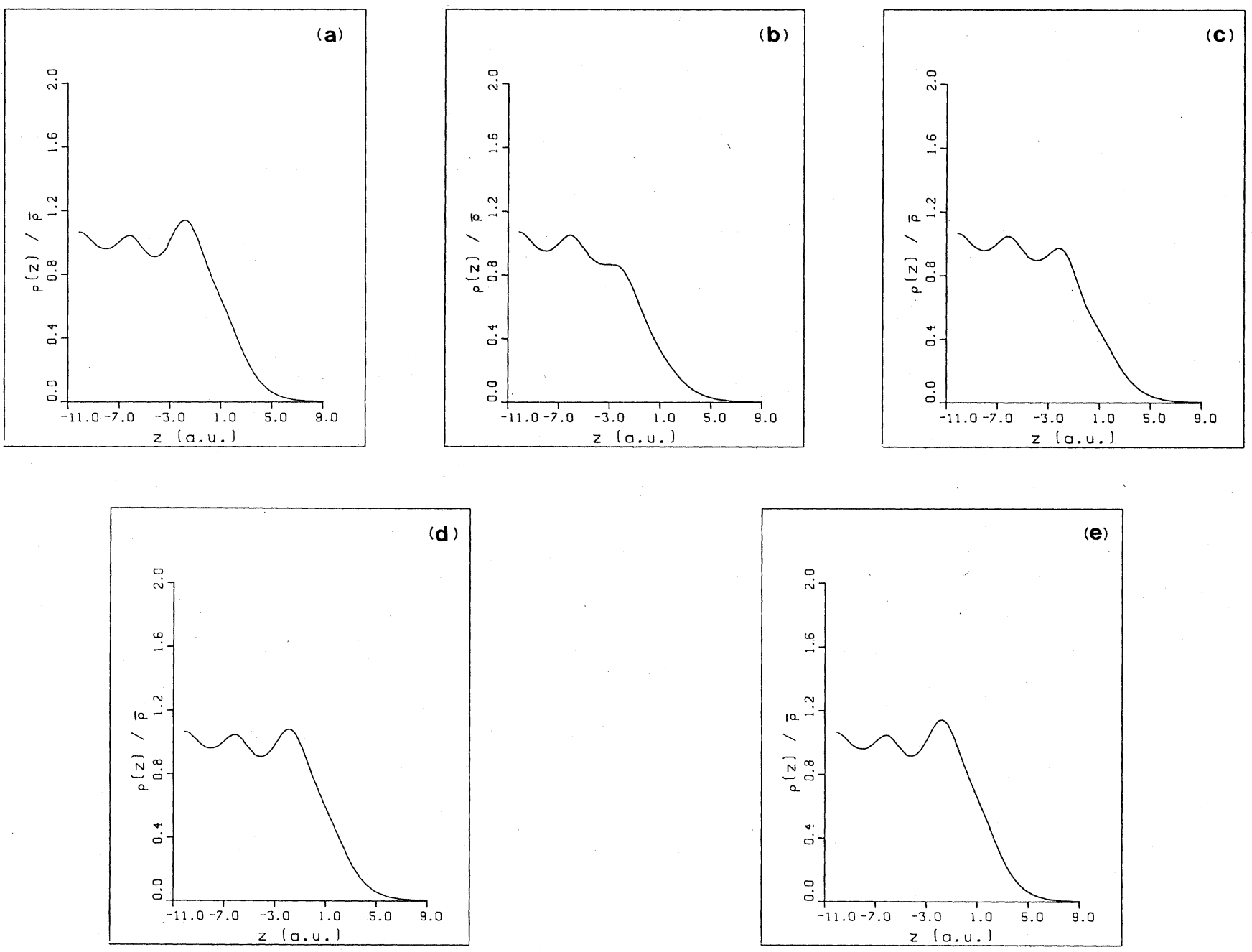

FIG. 3. (a)-(e) Electron density profiles $\rho(z)$ obtained self-consistently for the free $\mathrm{Na}(001)$ crystal and the one on which a new layer at different ionic concentrations is built on top of the free surface layer. The concentration is expressed in terms of $Z_{\text {eff }}$ (see text). The electron density is given in units of the average value $\bar{\rho}$ of $\rho(z)$ over a period along the $z$ axis in the bulk crystal. The surface plane is located at $z=0.0$. Figure 3(a) refers to the free $\mathrm{Na}(001)$ crystal. Figures $3(\mathrm{~b})-3(\mathrm{e})$ refer to the case where a new surface layer with ionic concentration $Z_{\text {eff }}=0.5,0.7,0.9$, and 1.0 , respectively, is built up on top of the surface layer of the free $\mathrm{Na}(001)$ crystal. 


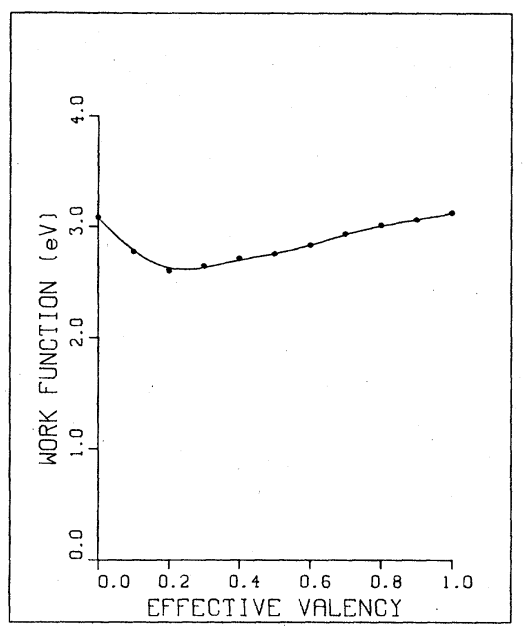

FIG. 4. Work function (in $\mathrm{eV}$ ) of the $\mathrm{Na}(001)$ semi-infinite crystal as a function of the concentration of the positive ions on the surface plane. The concentration is expressed in terms of the effective valency $Z_{\text {eff }}$ given by Eq. (5) of text. These results (solid circles) are obtained self-consistently using the zerothorder model approximation.

shows how the work function of $\mathrm{Na}(001)$ is changed as a new lattice plane is built on top of its surface in the case where no relaxation effects are present.

The self-consistency achieved in these calculations allows us to to define the average value of the effective single-electron potential far from the surface, inside the crystal with an error of $\pm 0.05 \mathrm{eV}$. At self-consistency, the charge neutrality is smaller than $0.5 \%$ for a total negative charge of three unit cells of each crystal. A small numerical error may be associated with a slight difference in the two bulk peaks (the two left peaks in the figures) of the electron density profiles.

For the perfect $\mathrm{Na}(001)$ crystal our computed work function is $3.08+0.05 \mathrm{eV}$ which is slightly higher than our previously ${ }^{16}$ reported value of $3.03 \pm 0.05 \mathrm{eV}$. The calculated value for the work function of the $\mathrm{Na}(001)$ crystal, on the surface of which a complete lattice plane is built, is $3.12 \mathrm{eV}$. The slightly higher value of the work function of the $\mathrm{Na}(001)$ crystal with one layer built in its surface may be attributed either to numerical errors associated with an increase in the integration regions in the cases where a new plane is built, or to the small increase in the peak values of the electron density profile in the bulk region. These errors, however, are within our $\pm 0.05 \mathrm{eV}$ allowance in the average value of the single-electron effective potential during the process to self-consistency. It may be claimed therefore that our presented results indicate quite accurately (and to our knowledge for the first time) the changes in a surface (electronic) property which take place during the process of building up a new lattice plane on a metallic surface. The comparison of the electron density profiles of Figs. 3(a) and 3(e) which corre- spond to a perfect $\mathrm{Na}(001)$ crystal and another (perfect) $\mathrm{Na}(001)$ crystal, which resulted after having built up a complete lattice plane on its surface, indicates the significance of the model calculations which incorporate lattice effects within the zeroth-order approximation explained above.

The variation of the work function with surface completeness exhibits, as expected, similarities with the workfunction changes due to ionic chemisorption. ${ }^{13} \mathrm{~A}$ deep minimum of $0.5 \mathrm{eV}$ is observed when the surface plane has ionic concentration $20-25 \%$ of that of a corresponding complete lattice plane. As the ionic concentration of the surface plane is increased the work function increases to reach finally the value of a perfect surface. In the case of ionic chemisorption, the variation of the work function is associated with the changes in the electrostatic surface dipole moment as the total charge distribution is changing in the surface region. At a concentration of a full monolayer, the work function of the chemisorbed system usually reaches the value of the chemisorbed (metallic) species.

The above explanation for the variation of the work function due to ionic chemisorption of an absorbate (which is either of the same or different type from the substrate) is based on the work-function definition ${ }^{3}$ (at zero temperature)

$$
\phi=V_{\text {eff }}(z=+\infty)-\left\langle V_{\text {eff }}(-\infty)\right\rangle-E_{F},
$$

which defines the work function $\phi$ (in the local singleelectron approximation) as the energy difference of an electron with kinetic energy $E_{F}$ far from the surface inside the metal (at $z=-\infty$ ) and of an electron at rest in the vacuum region (at $z=+\infty$ ). In Eq. (6), $V_{\text {eff }}(z)$ $=V_{0}(z)+V_{\mathrm{xc}}(z)$ and $V_{\text {eff }}(z=+\infty)$ is taken as the zerolevel energy.

However, when high concentrations of the adsorbates are involved, it is observed that the electron density profiles shown in Figs. 3(a)-3(c) exhibit a small region next to the tail region where the average electron density is smaller than its corresponding value deeply inside the crystal. This structure of the electron density profile is reflected in the single-electron potential. Thus it may be argued about the appropriate average value of the effective potential inside the bulk crystal (at $z=-\infty$ ) which must be used with $\mathrm{Eq}$. (6). This question raises the problem of inadequacy of the definition (6) and/or its nonlocal generalization. ${ }^{28}$ This question becomes more clear in the case of chemisorbed systems. These systems exhibit the work function of the absorbate when a complete chemisorbed layer is formed on the surface of the substrate. It should be more reasonable for these systems to use the definition for the work function, ${ }^{29}$

$$
\phi=\left[E_{N-1}+V_{\text {eff }}(z=+\infty)\right]-E_{N},
$$

where $E_{N}$ denotes the total energy of a system of $N$ electrons. The definition of Eq. (7) is equivalent to the definition of Eq. (6). Equation (7), however, is more difficult to use in numerical applications than Eq. (6). Thus Eq. (6) is of extensive use in the work-function calculations.

We have also used Eq. (6) in the calculation of the work function. Thus, it is expected, the value for the work function we find (in the case where the surface plane is an 
incomplete lattice plane with ionic concentration more than $50 \%$ (of that of a complete lattice plane) to appear slightly greater than the ones which would have been found using Eq. (7). For concentrations less than $50 \%$ our reported values are expected to be consistent with both equations (6) and (7). This expectation is justified from the fact that our computed electron density profiles show (for concentrations less than 50\%) a small shoulder (near the position of the chemisorbed plane) which disturbs slightly the tail region of the profile.
In concluding, we note that the zeroth-order model approximation proved very useful in following the workfunction changes during the process of building up a new lattice plane on top of the surface plane. The advantage of the present approach is that it incorporates bandstructure effects in a direct way within its self-consistent scheme. Relaxation effects may be easily incorporated. Finally, this method, since it is the zeroth-order approximation of a general three-dimensional theory, can be easily extended so as to include the lattice effects completely.
1J. Appelbaum and D. R. Hamann, Phys. Rev. B 6, 2166 (1972).

${ }^{2}$ N. D. Lang and W. Kohn, Phys. Rev. B 1, 4555 (1970).

${ }^{3}$ N. D. Lang and W. Kohn, Phys. Rev. B 3, 1215 (1971).

${ }^{4}$ R. Monnier and J. P. Perdew, Phys. Rev. B 17, 2595 (1978).

${ }^{5}$ R. Monnier, J. P. Perdew, D. C. Langreth, and J. W. Wilkins, Phys. Rev. B 18, 656 (1978).

${ }^{6}$ F. Jona, D. Sondericker, and P. M. Marcus, J. Phys. C 13, L155 (1980).

${ }^{7}$ A. Bianconi and R. Z. Bachrach, Phys. Rev. Lett. 19, 104 (1979).

${ }^{8}$ K. P. Bohnen, Surf. Sci. 115, L96 (1982).

${ }^{9}$ J. N. Andersen, H. B. Nielsen, L. Petersen, and D. L. Adams, J. Phys. C 17, 173 (1984).

${ }^{10}$ R. N. Barnett, U. Landman, and C. L. Cleveland, Phys. Rev. B 28, 1685 (1983).

${ }^{11}$ K. F. Wojciechowski, Surf. Sci. 80, 253 (1979).

12J. P. Perdew, Phys. Rev. B 25, 6291 (1982).

${ }^{13}$ N. D. Lang, Phys. Rev. B 4, 4234 (1971).

${ }^{14}$ A. Kiejna and K. F. Wojciechowski, Solid State Commun. 31, 857 (1979).

${ }^{15}$ K. P. Bohnen and S. C. Ying, Phys. Rev. B 22, 1806 (1980).
${ }^{16}$ A. N. Andriotis, Surf. Sci. 116, 501 (1982).

${ }^{17}$ A. N. Andriotis and C. A. Nicolaides, Surf. Sci. 116, 513 (1982).

${ }^{18}$ D. W. Jepsen and P. M. Marcus, in Computational Methods in Band Theory, edited by P. M. Marcus, J. F. Janak, and A. R. Williams (Plenum, New York, 1971).

${ }^{19}$ P. Hohenberg and W. Kohn, Phys. Rev. 136, B864 (1964).

${ }^{20}$ A. N. Andriotis, Surf. Sci. 138, 269 (1984).

${ }^{21}$ N. W. Ashcroft, Phys. Lett. 23, 48 (1966).

22E. P. Wigner, Phys. Rev. 46, 1002 (1934).

${ }^{23}$ A. Kiejna, J. Phys. C 15, 4717 (1982).

${ }^{24}$ A. Kiejna and K. F. Wojciechowski, J. Phys. C 16, 6883 (1983).

${ }^{25}$ S. Anderson, J. B. Pendry, and P. M. Echenique, Surf. Sci. 65, 539 (1977).

${ }^{26}$ C. A. Nicolaides and A. N. Andriotis, Solid State Commun. 44, 99 (1982).

${ }^{27}$ C. A. Nicolaides and A. N. Andriotis, Int. J. Quantum Chem. 23, 561 (1983).

28J. Bardeen, Phys. Rev. 49, 653 (1936).

${ }^{29}$ F. K. Schulte, Z. Phys. B 27, 303 (1977). 